\title{
Erratum: More nonperturbative corrections to the fine and hyperfine splitting in heavy quarkonium \\ [Phys. Rev. D 55, 407 (1997)]
}

\author{
A. Pineda \\ (Published 16 March 1999)
}

[S0556-2821(99)02409-1]

PACS number(s): 14.40.Gx, 12.38.Lg, 99.10.+g

In Eq. (1.6) the factor 2 has to be replaced by a factor 4.

In the octet hyperfine potential one contribution was omitted. This new contribution is due to the tree level annihilation diagram which, unlike the singlet case, gives a nonzero contribution. This new contribution reads

$$
V_{8}^{\mathrm{HF}}(\text { annihilation })=\frac{4 N_{c}^{2}-3}{4 N_{c}^{2}} \frac{2 \pi \alpha_{s}}{m^{2}} \delta^{3}(\mathbf{r}) \mathbf{S}^{2} .
$$

Accordingly Eqs. (1.7), (3.1), (3.2), and (3.6) now read

$$
\begin{gathered}
V_{8}^{\mathrm{HF}}=\left(1-\frac{1}{3 N_{c}}-\frac{3}{4 N_{c}^{2}}\right) \frac{2 \pi \alpha_{s}}{m^{2}} \delta^{3}(\mathbf{r}) \mathbf{S}^{2}, \\
\delta E_{8}^{\mathrm{HF}}=-s(s+1) \delta_{l, 1}\left(1-\frac{1}{3 N_{c}}-\frac{3}{4 N_{c}^{2}}\right) \frac{\alpha_{s}}{6 m^{2}} \frac{\pi\left\langle\alpha_{s} G^{2}\right\rangle}{6 N_{c}}\left|\left\langle R_{n l}\left|r \frac{1}{E_{n}-H_{8}^{\left(l_{1}=0\right)}}\right| r=0\right\rangle\right|^{2}, \\
\delta E_{8}^{\mathrm{HF}}=-\delta_{l, 1} s(s+1) \frac{29}{4} \alpha_{s} \frac{\pi\left\langle\alpha_{s} G^{2}\right\rangle}{\left(m \tilde{\alpha}_{s}\right)^{3}} h f[n], \\
\Delta_{\mathrm{HF}}(\text { new })=\overline{\delta E_{8}^{F}}+\delta E_{8}^{\mathrm{HF}}=-\frac{\alpha_{s}}{\widetilde{\alpha}_{s}} \frac{\pi\left\langle\alpha_{s} G^{2}\right\rangle}{m^{3}\left(C_{f} \widetilde{\alpha}_{s}\right)^{2}} \frac{929717296}{18692671725} .
\end{gathered}
$$

The sentence after Eq. (3.6) should be replaced by the following: "which turns out to be around 5\% smaller." Note that this new contribution is $N_{c}$ enhanced with respect to the contributions already considered in the paper. 